\title{
Effect of sinomenine hydrochloride on radiosensitivity of esophageal squamous cell carcinoma cells
}

\author{
SHENBO FU ${ }^{1}$, LONG JIN ${ }^{2}$, TUOTUO GONG ${ }^{1}$, SHUPEI PAN ${ }^{1}$, SHUYU ZHENG ${ }^{1}$, XUANWEI ZHANG ${ }^{1}$, \\ TIAN YANG ${ }^{1}$, YUCHEN SUN ${ }^{1}$, YA WANG $^{1}$, JIA GUO $^{1}$, BEINA HUI $^{1}$ and XIAOZHI ZHANG ${ }^{1}$ \\ ${ }^{1}$ Department of Radiation Oncology, The First Affiliated Hospital of Xi'an Jiaotong University, Xi'an, Shaanxi 710061; \\ ${ }^{2}$ Department of Radiation Oncology, Shaanxi Province People's Hospital, Xi'an, Shaanxi 710068, P.R. China
}

Received September 8, 2017; Accepted January 17, 2018

DOI: $10.3892 /$ or.2018.6228

\begin{abstract}
Radiation therapy is one of the most important treatments for unresectable and locally advanced esophageal squamous cell carcinoma (ESCC), however, the response to radiotherapy is sometimes limited by the development of radioresistance. Sinomenine hydrochloride $(\mathrm{SH})$ has anticancer activity, but its effect on the radiosensitivity of ESCC is unclear. We determined the effect of $\mathrm{SH}$ on the radiosensitivity of ESCC cells and elucidated its potential radiosensitization mechanisms in vitro and in vivo. ESCC cells were subjected to $\mathrm{SH}$ and radiation, both separately and in combination. Untreated cells served as controls. The CCK-8 assay was used to evaluate cell proliferation, and the clonogenic assay to estimate radiosensitization. Flow cytometry was used to investigate cell cycle phases and cell apoptosis. Bcl-2, Bax, cyclin B1, CDK1, Ku86, Ku70, and Rad51 expression was evaluated using western blotting. In vivo, tumor xenografts were created using BALB/c nude mice. Tumor-growth inhibition was recorded, and $\mathrm{Ki}-67$ and Bax expression in the tumor tissues was assessed using immunohistochemistry. SH inhibited ESCC cell growth and markedly increased their radiosensitivity by inducing $\mathrm{G} 2 / \mathrm{M}$ phase arrest. SH combined with radiation therapy significantly increased ESCC cell apoptosis. The molecular mechanism by which $\mathrm{SH}$ enhanced radiosensitivity of ESCC cells was related to Bcl-2, cyclin B1, CDK1, Ku86, Ku70, and Rad51 downregulation and Bax protein expression upregulation. $\mathrm{SH}$ combined with radiation considerably delayed the growth of tumor xenografts in vivo. Immunohistochemical analysis showed that in the $\mathrm{SH}$ combined with radiation group, the expression of Bax was significantly higher while that of Ki-67 was lower than the expressions in the control groups. Taken together, our findings
\end{abstract}

Correspondence to: Professor Xiaozhi Zhang, Department of Radiation Oncology, The First Affiliated Hospital of Xi'an Jiaotong University, Yanta West Road 277, Xi'an, Shaanxi 710061, P.R. China E-mail: zhangxiaozhi@xjtu.edu.cn

Key words: sinomenine hydrochloride, radiosensitivity, esophageal squamous cell carcinoma, cell cycle, apoptosis, DNA repair showed that $\mathrm{SH}$ could improve the sensitivity of radiation in ESCC cells by inducing G2/M phase arrest, promoting radiation-induced apoptosis and inhibiting DSB-repair pathways. $\mathrm{SH}$ appears to be a prospective radiosensitizer for improving the efficacy of radiotherapy for ESCC.

\section{Introduction}

Esophageal cancer, a highly malignant cancer, is the sixth leading cause of cancer deaths in the world and has a 5-year survival rate of less than $25 \%(1,2)$. Esophageal adenocarcinoma and esophageal squamous cell carcinoma (ESCC) are the two main types of esophageal cancer. In Asia and Northern Iran, ESCC is the most common histological subtype of esophageal cancer and accounts for $90 \%$ of all esophageal cancer patients (3). Radiotherapy plays a crucial part in the management of patients with inoperable and locally advanced ESCC. Unfortunately, radioresistance results in local cancer recurrence and poor prognosis in ESCC patients (4). Thus, the development of novel radiosensitizing agents, which can enhance the response of cancer cells to radiation and improve the survival of patients with radioresistant ESCC is clinically warranted and significant. Although several potential radiosensitizers of ESCC have been investigated, a satisfactory agent has not yet been discovered.

The radiosensitivity of cancer cells depends on multiple factors, including the regulation of the cell cycle, apoptosis, and interference with DNA-repair pathways. Studies have shown that cells are most radiosensitive in the G2/M phase and least radiosensitive in the $S$ phase. Several radiosensitizers were found to possess the ability to regulate the cell cycle and result in arrest at the G2/M stage $(5,6)$. The apoptotic pathway is involved in tumor cell survival after radiation. Thus, radiosensitivity can also be increased by promoting radiation-induced apoptosis of cancer cells (7). In addition, one of the most pivotal mechanisms of radiation-induced cancer cell death is DNA damage, especially DNA double-strand breaks (DSBs). Inhibition of the repair of radiation-induced DSBs has been demonstrated to increase the radiosensitivity of cancer cells $(8,9)$.

Several traditional Chinese medicines with antitumor properties and only few side effects have been studied. Sinomenine is an alkaloid extracted from the traditional Chinese herb 
Sinomenium acutum. Sinomenine has pharmacologically relevant properties such as anti-arthritic (10), anti-inflammatory (11), analgesic (12), and immunosuppressive effects (13). Sinomenine hydrochloride (SH) has been effectively used to treat rheumatoid arthritis in clinical practice (14). More recently, several studies have demonstrated that SH has antineoplastic effects against various types of cancer, including lung cancer (15), hepatic cancer (16), breast cancer (17), osteosarcoma (18), and colon cancer (19). The anticancer effects of SH include anti-metastasis, anti-angiogenesis, anti-proliferation, and apoptosis induction. Furthermore, it has been found that SH inhibits the proliferation of ESCC cell line Eca109, promotes apoptosis, and significantly increases chemosensitivity of cancer cells to 5-fluorouracil (20). However, to our knowledge, no study has yet investigated the radiosensitizing effect of SH on ESCC. With this in mind, the present study was undertaken with a view to determining the effects of $\mathrm{SH}$ on the radiosensitivity of ESCC cells and clarifying the molecular mechanisms underpinning these effects.

\section{Materials and methods}

Cell culture and chemicals. Eca109 and EC9706, two human ESCC cell lines, were obtained from the Chinese Academy of Sciences Cell Bank (Shanghai, China). Dulbecco modified Eagle medium (DMEM; Gibco, Carlsbad, CA, USA) supplemented with 10\% fetal bovine serum (Sijichun Bioengineering Materials Inc., Zhejiang, China) was used as the cell culture medium. For some studies, SH (Sigma-Aldrich Co., St. Louis, MO, USA) was dissolved in DMEM to achieve a concentration of $10 \mathrm{mM}$. Cell cultures were housed at $37^{\circ} \mathrm{C}$ in a humidified incubator with 5\% $\mathrm{CO}_{2}$. The CCK-8 kits and cell cycle analysis kits were acquired from Beyotime Institute of Biotechnology (Jiangsu, China). Annexin V-7AAD apoptosis-detection kits were purchased from BD Biosciences (Franklin Lakes, NJ, USA).

Cell proliferation assay. ESCC cell viability was assessed using CCK-8 assays. Cells were placed in 96 -well plates $\left(3 \times 10^{3}\right.$ cells/well) and then exposed to $0,0.04,0.4,1,2.5$, or $5 \mathrm{mM} \mathrm{SH}$ for 24,48 , or $72 \mathrm{~h}$. In the combination treatment group, cells were pretreated with $\mathrm{SH}$ for $24 \mathrm{~h}$, and X-rays (8 Gy) for another $24 \mathrm{~h}$. After the treatments, $10 \mu \mathrm{l} \mathrm{CCK}-8$ was added, and the incubation was continued for another $4 \mathrm{~h}$ at $37^{\circ} \mathrm{C}$. We then measured the absorbance of each well at $450 \mathrm{~nm}$ by using spectrophotometry. The procedure was performed in triplicate, and average values are reported.

Clonogenic assay. Cells were pretreated with or without $\mathrm{SH}$ for $48 \mathrm{~h}$, then seeded in 6-well plates and irradiated at $0,2,4$, 6 , or 8 Gy $(200,200,600,1,000$ or 3,000 cells per well) with 4-MV X-rays by using a linear accelerator (Simens, Munich, Germany). After incubation for 14 days at $37^{\circ} \mathrm{C}$ with $5 \% \mathrm{CO}_{2}$, the colonies formed were washed with phosphate-buffered saline (PBS), fixed with methanol, and stained with Giemsa. Only colonies containing more than 50 cells were counted. The experiment was performed in triplicate. The survival curves were fitted using the single-hit multi-target model in GraphPad Prism 5 (GraphPad Software Inc., La Jolla, CA, USA). Then, the D0 (mean lethal dose), Dq (quai-threshold),
Table I. The properties of a multi-target model in ESCC cells as assessed through the clonogenic assay.

\begin{tabular}{lcccc}
\hline Cell line & D0 & Dq & SF2 & SER \\
\hline Eca109 & 2.43 & 2.07 & 0.73 & 1.80 \\
Eca109+SH & 1.35 & 1.61 & 0.57 & \\
EC9706 & 2.17 & 2.39 & 0.74 & 1.54 \\
EC9706+SH & 1.41 & 1.17 & 0.47 & \\
\hline
\end{tabular}

D0, mean lethal dose; Dq, quai-theshold; SF2, survival fraction at $2 \mathrm{~Gy}$; SER, sensitizing enhancement ratio.

and SF2 (survival fraction at $2 \mathrm{~Gy}$ ) were obtained based on the clonogenic assay. The sensitizing enhancement ratio (SER) was calculated as the ratio of D0-control cells to D0-SH-treated cells. The interaction between $\mathrm{SH}$ and radiation was examined using the combination index (CI) method of Chou and Talalay (21) and CompuSyn software (Biosoft, Cambridge, $\mathrm{UK}) . \mathrm{CI}<1$ indicates synergic effect, $\mathrm{CI}=1$ indicates additive effect, and $\mathrm{CI}>1$ indicates antagonistic effect.

Cell cycle analysis. ESCC cells were seeded in 6-well plates at a density of $2 \times 10^{5}$ cells per well and divided into four groups: Control group, $\mathrm{SH}$ group, radiation group, and $\mathrm{SH}+$ radiation group. In the SH group, cells were exposed to $\mathrm{SH}$ for $48 \mathrm{~h}$, and in the radiation group, cells were irradiated with $8 \mathrm{~Gy}$ $\mathrm{X}$-rays for $24 \mathrm{~h}$. In the combination treatment group, cells were pretreated with $\mathrm{SH}$ for $24 \mathrm{~h}$ and then irradiated at $8 \mathrm{~Gy}$ for another $24 \mathrm{~h}$. After the treatments, at least $1 \times 10^{6}$ cells were collected, fixed with $70 \%$ ethanol $\left(2 \mathrm{~h}, 4^{\circ} \mathrm{C}\right)$, and stained with propidium iodide and RNase $\mathrm{A}\left(30 \mathrm{~min}, 37^{\circ} \mathrm{C}\right)$. We performed cell cycle analysis by using flow cytometry (BD Biosciences).

Apoptosis assay. ESCC cells were divided into the following experimental groups: $\mathrm{SH}$ group, radiation group, $\mathrm{SH}+$ radiation group, and control group. ESCC cells $\left(2 \times 10^{5}\right.$ cells/well $)$ were seeded in 6-well plates and pretreated in the same way as they were for the cell cycle analyses. Apoptosis was measured using flow cytometry and the Annexin V-7AAD apoptosis-detection kit. At least $1 \times 10^{6}$ cells were incubated at $4^{\circ} \mathrm{C}$ with propidium iodide and Annexin V-7AAD, and the percentage of apoptotic cells was calculated using flow cytometry (BD Biosciences).

Western blot analysis. Cells were lysed with radioimmunoprecipitation assay lysis and extraction buffers (Pioneer Technology, Xi'an, China), separated using sodium dodecyl sulfate polyacrylamide gel electrophoresis, and then transferred to polyvinylidene difluoride membranes (Millipore, Billerica, MA, USA). The membranes were incubated at $4^{\circ} \mathrm{C}$ with the following primary antibodies: Anti-Bax (sc-20067, 1:1,000), anti-Bcl-2 (sc-509, 1:1,000), anti-Ku86 (sc-5280, 1:500), anti-Ku70 (sc-17789, 1:1,000), anti-Rad51 (sc-133089, 1:500), anti-cyclin B1 (sc-7393, 1:1,000), anti-CDK1 (sc-53219, 1:500), (all from Santa Cruz Biotechnology, Santa Cruz, CA, USA), and anti-GAPDH (\#5174, 1:3,000; Cell Signaling Technology, Inc., Danvers, MA, USA). Then incubated with 

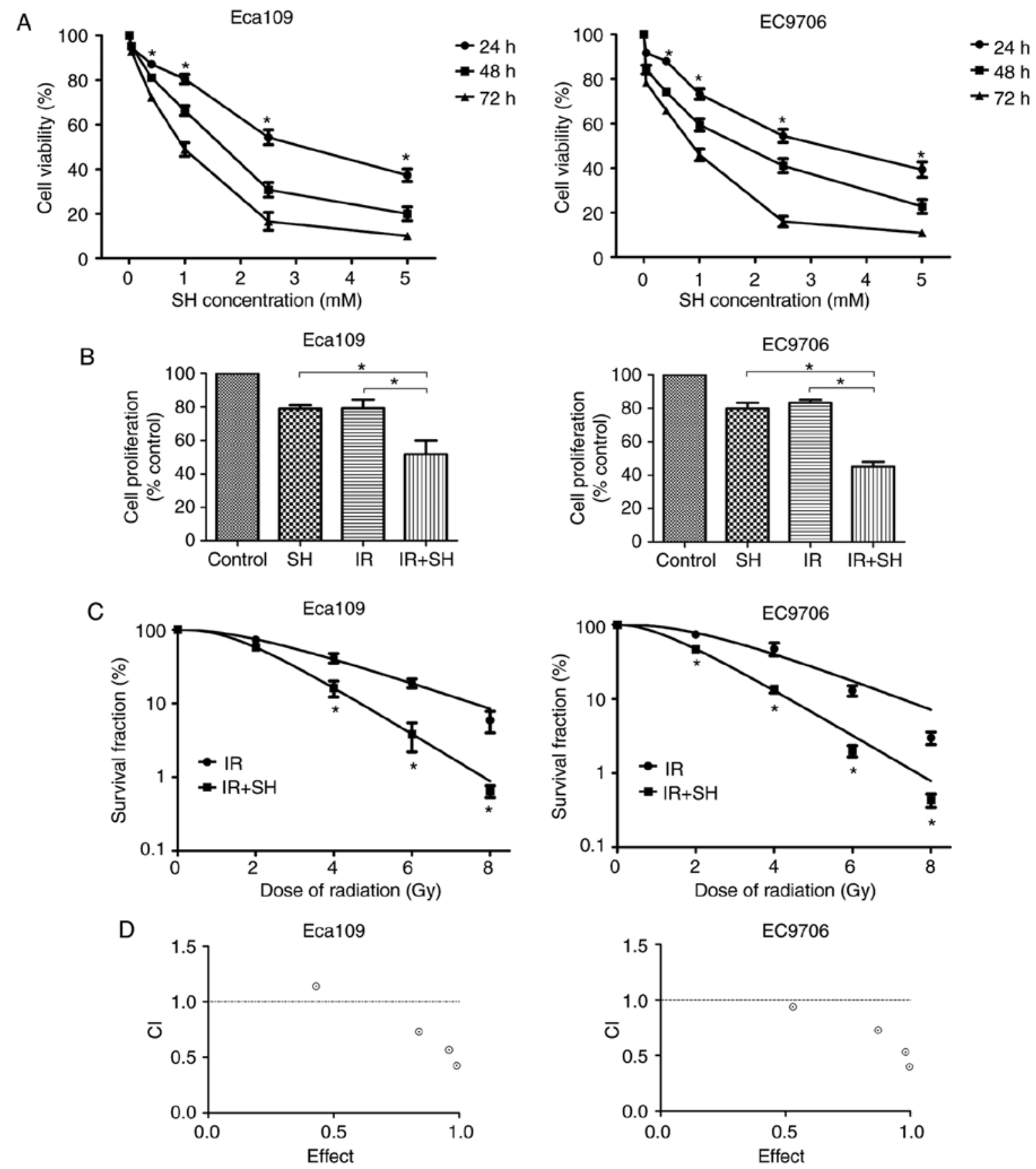

Figure 1. SH enhances the radiosensitivity of ESCC cells. (A) Eca109 and EC9706 cells were treated with SH $(0,0.04,0.4,1,2.5$, or $5 \mathrm{mM})$ for 24,48 , or $72 \mathrm{~h}$, after which cell viability was evaluated using the CCK-8 assay. (B) Cells were pretreated with SH (0.3 mM for Eca109 and $0.4 \mathrm{mM}$ for EC9706) and/or exposed to 8 Gy X-rays, and then analyzed using the CCK-8 assay. (C) Cells were pretreated with SH and exposed to 0,2, 4, 6, or 8 Gy X-rays. After 14 days, colonies were stained and counted. The survival curve was obtained using the multi-target model. (D) The interaction between SH and radiation was examined using the combination index $(\mathrm{CI})$ method of Chou and Talalay and CompuSyn software. $\mathrm{CI}=1$, additive effect, $\mathrm{CI}<1$, synergism, CI $>1$, antagonism (" $\mathrm{P}<0.05$ ).

secondary antibodies coupled with horseradish peroxidase at room temperature for $1.5 \mathrm{~h}$, anti-mouse $(\# 4410,1: 5,000)$ or anti-rabbit (\#4414, 1:5,000) antibodies (Cell Signaling Technology, Inc.). The membranes were visualized using a chemiluminescence reagent (Millipore) and the ChemiDoc System (Bio-Rad, Hercules, CA, USA).

Xenograft tumor model. The animal experiments were approved by the institutional animal Ethics Committee of the First Affiliated Hospital of Xi'an Jiaotong University. The experimental protocol complied with the animal ethics guidelines of the First Affiliated Hospital of Xi'an Jiaotong
University. Sixteen mice were housed in sterile cages under standard condition (12-h light/dark cycles at $21 \pm 2^{\circ} \mathrm{C}$ ) with ad libitum access to disinfected water and food. AnESCC model was established in female BALB/c nude mice (aged 4 weeks; Experimental Animal Center, Xi'an Jiaotong University) by injecting $5 \times 10^{6}$ Eca109 cells subcutaneously into the backs of the mice. When the tumor volume was $100 \mathrm{~mm}^{3}$, the animals were randomly assigned to the following groups $(n=4$ per group): radiation, $\mathrm{SH}$, radiation $+\mathrm{SH}$, and control groups. $\mathrm{SH}$ was intraperitoneally injected at a dose of $75 \mathrm{mg} / \mathrm{kg}$, once daily for 7 days. Tumors were treated with $4 \mathrm{~Gy} \mathrm{X}$-rays for 3 consecutive days (total dose, $12 \mathrm{~Gy}$ ), starting from the second 

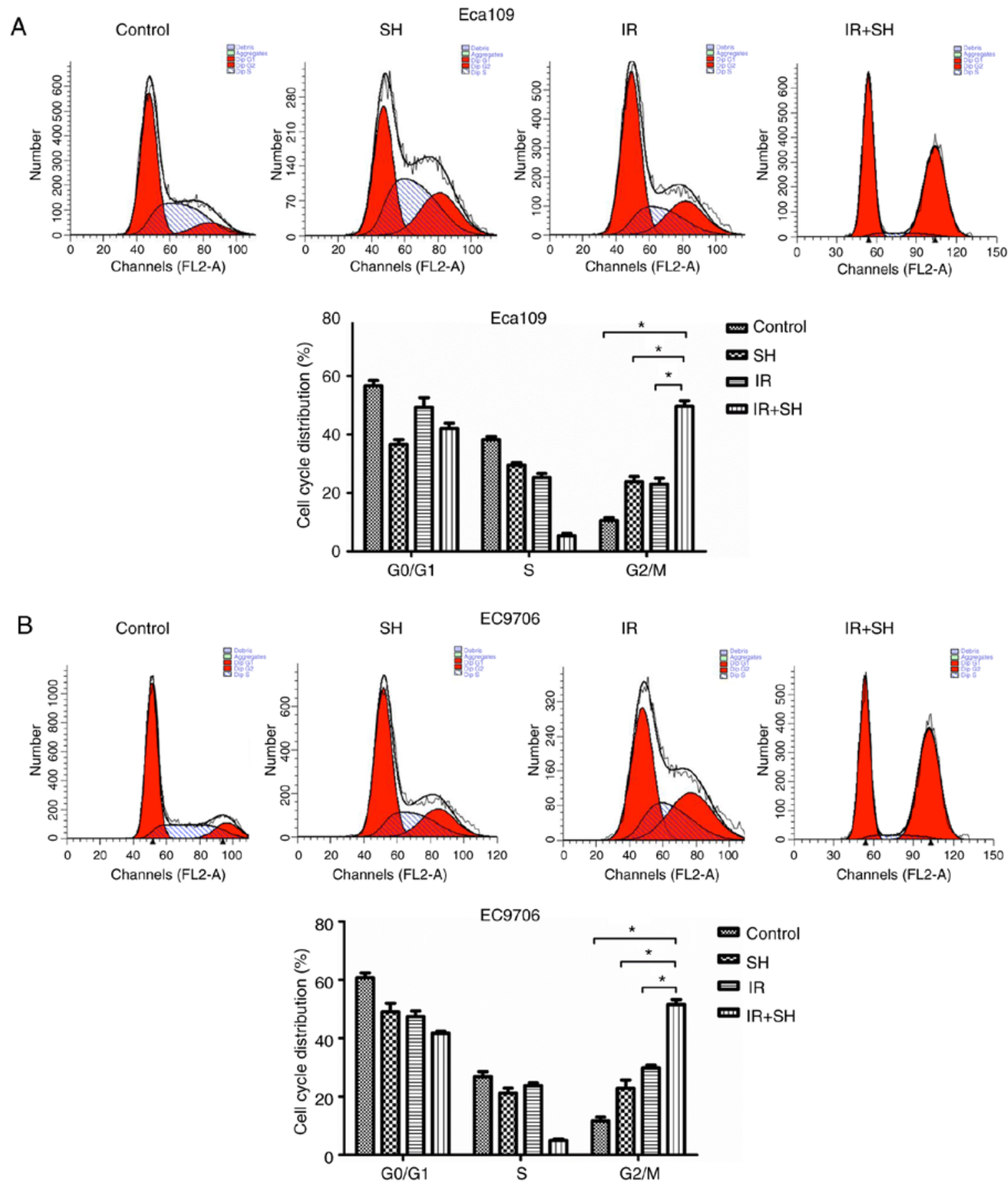

Figure 2. Cell cycle changes induced by SH and radiation in ESCC cells. Cells were pretreated with SH and/or exposed to 8 Gy X-rays, and then analyzed using flow cytometry. Combined treatment with SH and radiation significantly induced G2/M-phase arrest in ESCC cells ("P<0.05). (A) Eca109, (B) EC9706.

day of drug administration. The mice in the control group were intraperitoneally inoculated with equal volumes of PBS. Mouse body weight and tumor volume (length $\mathrm{x}$ width ${ }^{2} \mathrm{x} 0.5$ ) were measured using calipers every 3 days for 30 days. All mice were sacrificed using pentobarbital sodium at a dose of $100 \mathrm{mg} / \mathrm{kg}$ after 30 days, and the tumors were harvested.

Immunohistochemistry. Tumor tissue samples were fixed with $10 \%$ formalin, paraffin embedded, and then stained with hematoxylin-eosin. Immunohistochemical staining was performed according to the standard protocol. Tumor-tissue sections were incubated overnight at $4^{\circ} \mathrm{C}$ with primary antibodies against
Ki-67 (sc-23900, 1:300; Santa Cruz Biotechnology) and Bax (\#5023, 1:300; Cell Signaling Technology, Inc.), and then with anti-mouse or anti-rabbit secondary antibodies for $1 \mathrm{~h}$. Finally, images were captured using microscopy, and five random fields were chosen in each specimen for analysis.

Statistical analysis. The data were expressed as mean \pm SEM. Statistical analysis was performed using Graphpad Prism 5. Differences between the control and treatment groups were tested using analysis of variance (ANOVA) followed by Bonferroni's post-hoc test. Differences were considered to be significant at $\mathrm{P}<0.05$. 
A
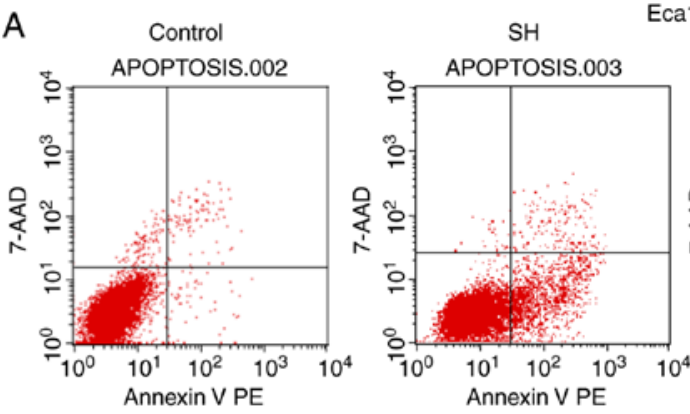

Eca109
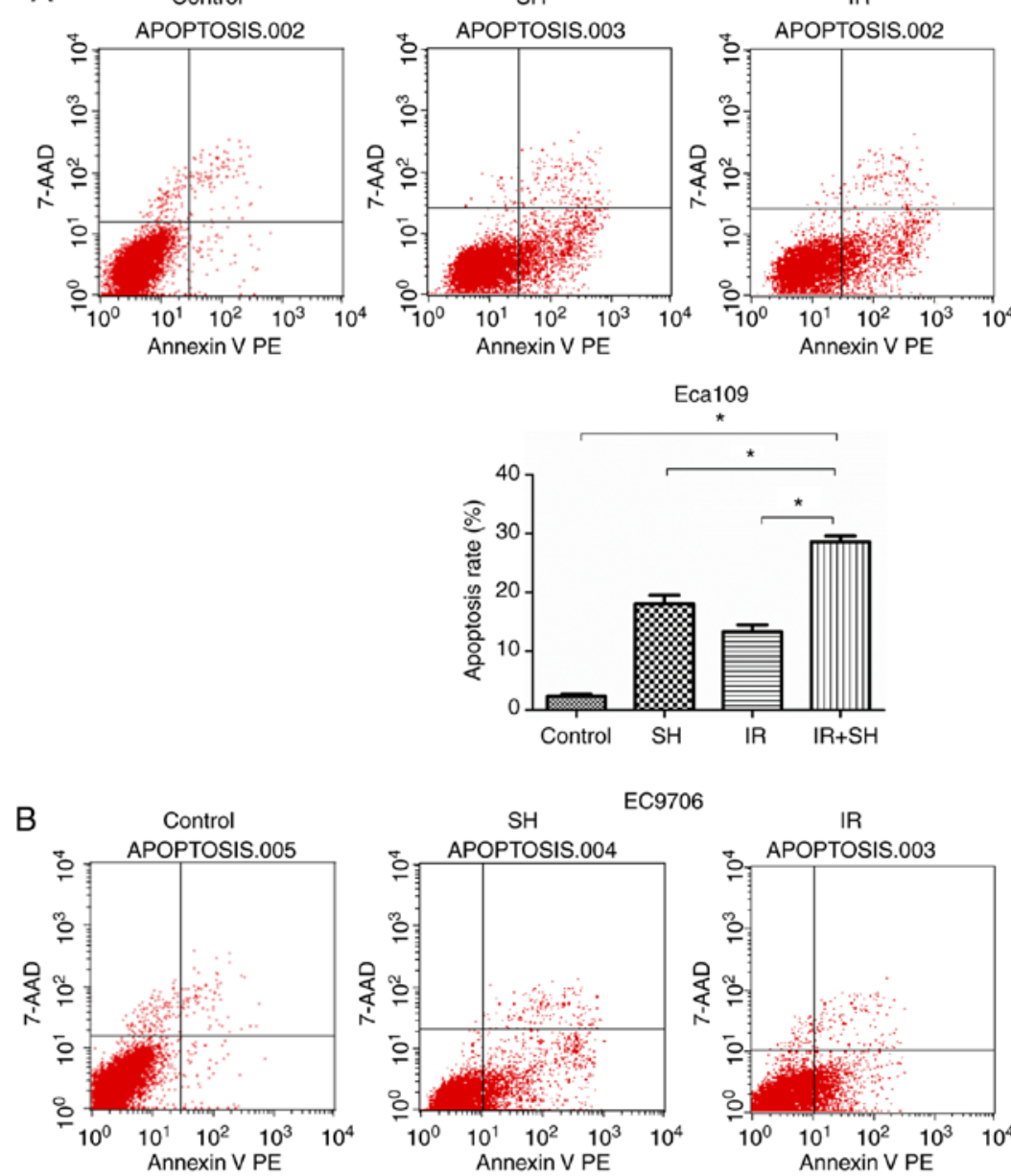

EC9706

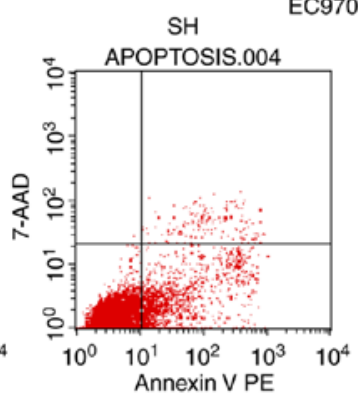

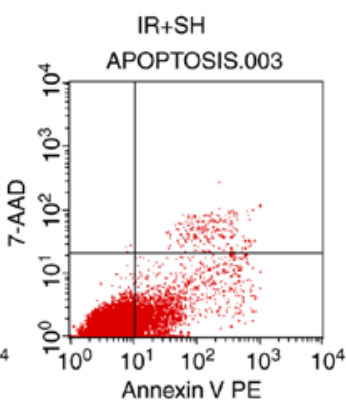

$\mathrm{IR}+\mathrm{SH}$
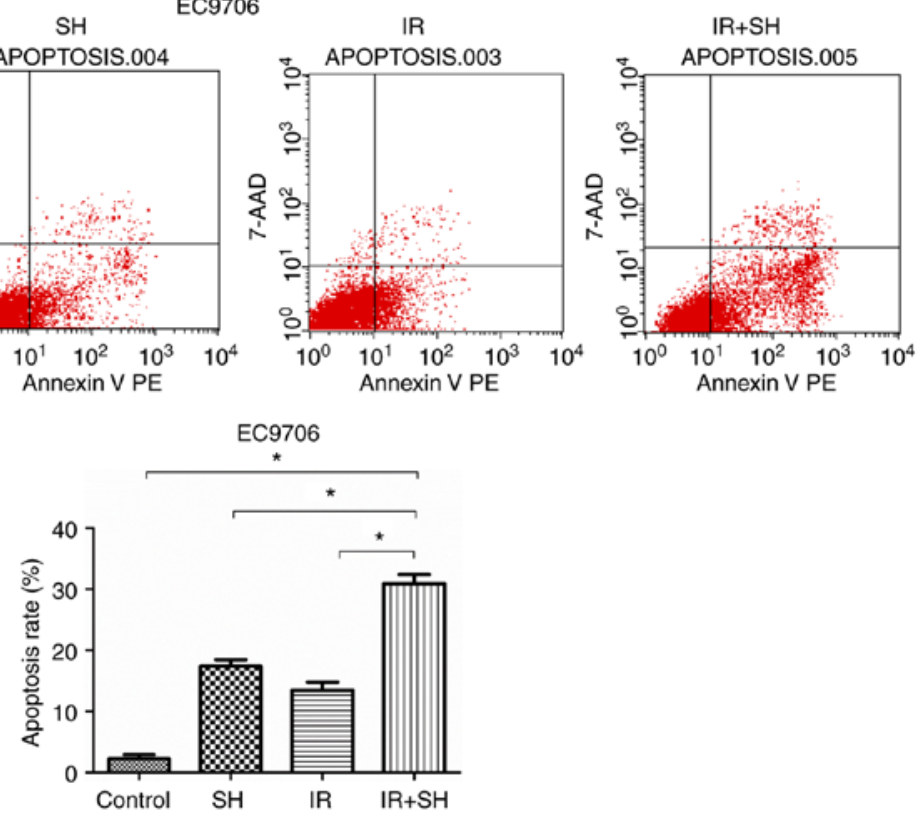

Figure 3. Effect of SH on ESCC cell apoptosis. ESCC cells were pretreated with SH and exposed to 8 Gy X-ray. Annexin V-PE/7AAD staining and flow cytometry were used to measure and analyze the cell apoptosis ratio. SH combined with radiation therapy significantly increased ESCC cell apoptosis $\left({ }^{*} \mathrm{P}<0.05\right)$. (A) Eca109, (B) EC9706.

\section{Results}

SH inhibits ESCC cell growth and enhances radiosensitivity of ESCC cells. To determine whether SH affected ESCC cell proliferation, we treated ESCC cells with various concentration of SH (0-5 mM) for 24-72 h. The CCK-8 assay was performed to estimate cell viability. The results showed that SH significantly inhibited ESCC cell viability in a time- and concentration-dependent manner $(\mathrm{P}<0.05$; Fig. 1A). In the case of the $48 \mathrm{~h}$ treatment period, the half-maximal inhibitory concentration $\left(\mathrm{IC}_{50}\right)$ of $\mathrm{SH}$ for Eca109 and EC9706 cells was 1.31 and $1.41 \mathrm{mM}$, respectively. We selected the $48 \mathrm{~h} \mathrm{IC}_{20}$ values $(0.3 \mathrm{mM}$ for Eca109 and $0.4 \mathrm{mM}$ for EC9706) as a appropriate concentration for the subsequent experiments. We then evaluated the inhibitory effects of $\mathrm{SH}$, radiation, and $\mathrm{SH}$ combined with radiation on the proliferation of ESCC cells. The CCK-8 assay showed that SH combined with radiation dramatically restrained ESCC cell proliferation compared with $\mathrm{SH}$ or radiation group $(\mathrm{P}<0.05$; Fig. 1B).

The radiosensitization effect of SH on ESCC cells was assessed using the clonogenic assay. The results showed that SH significantly improved the radiosensitivity of ESCC cells in comparison with the control group $(\mathrm{P}<0.05$; Fig. $1 \mathrm{C})$. We calculated the radiation parameters based on the results of the clonogenic survival assay. The properties of a multi-target model in ESCC cells are detailed in Table I. In the absence of SH, the SF2 in Eca109 and EC9706 cells was 0.73 and 0.74, while after treatment with $\mathrm{SH}$, the SF2 decreased to 0.57 and 
0.47 , respectively. The SER was 1.80 and 1.54 in Eca109 cells and EC9706 cells, respectively. CI values less than 1 indicated SH combined with radiation resulted in synergic effect (Fig. 1D). These results indicate that SH sensitized ESCC cells to radiotherapy.

SH causes G2/M phase arrest in ESCC cells. To observe the effect of SH on the cell cycle distribution of ESCC cells, we used flow cytometry. Cell cycle analysis demonstrated that $\mathrm{SH}$ combined with radiation arrested ESCC cells in the G2/M phase (Fig. 2). The number of cells in this phase was significantly higher in the combination treatment group than in the $\mathrm{SH}$, radiation, and control groups $(\mathrm{P}<0.05)$. Thus, $\mathrm{SH}$ enhanced the radiosensitivity of ESCC cells by increasing ratio of $\mathrm{G} 2 / \mathrm{M}$ phase cells.

SH induces apoptosis of ESCC cells. We performed Annexin V-PE/7AAD flow cytometry to measure the effect of $\mathrm{SH}$ on apoptosis of ESCC cells. We found that $\mathrm{SH}$ and radiation in combination increased the apoptosis ratio in ESCC cells as compared with the control, $\mathrm{SH}$ and radiation group $(\mathrm{P}<0.05$; Fig. 3). Based on this finding, SH increased radiosensitivity by promoting radiation-induced apoptosis of ESCC cells.

SH upregulates Bax and downregulates Bcl-2, cyclin B1, CDK1, Ku-70, Ku-86, and Rad51 in ESCC cells. To probe the molecular mechanism underpinning the effects of SH on the radiosensitivity of ESCC cells, we examined the expression of $\mathrm{G} 2 / \mathrm{M}$ phase-associated proteins, apoptotic proteins and DSB-repair proteins using western blot analysis. Our findings showed that the combination of SH and 8 Gy irradiation could upregulate the expression of Bax compared with the $\mathrm{SH}$ group and radiation group; conversely, the expression of $\mathrm{Bcl}-2$, cyclin B1, CDK1, Ku-70, Ku-86, and Rad51 was downregulated in the combination-treatment group compared with other three groups (Fig. 4).

Combined treatment with radiation and SH delays ESCC tumor growth in vivo. To determine whether $\mathrm{SH}$ affected the radiosensitivity of ESCC cells in vivo, an ESCC model was established by injecting Eca109 cells into nude mice. We found that SH could considerably inhibit tumor growth. The tumor volume was markedly smaller in the radiation $+\mathrm{SH}$ group than in the other three groups $(\mathrm{P}<0.05$; Fig. $5 \mathrm{~A})$. Furthermore, immunohistochemical analysis showed that in the combined treatment group, the expression of Bax was significantly upregulated while that of Ki-67 was downregulated as compared with the expressions in the other three groups (Fig. 5B).

\section{Discussion}

Radiotherapy is an effective therapeutic strategy for inoperable and locally advanced ESCC, although it is sometimes limited by the development of radioresistance (4). Thus, a radiosensitizer with a high benefit-to-risk ratio is required. $\mathrm{SH}$ has been reported to exhibit antitumor action by regulating cell proliferation, inhibiting metastasis, and inducing apoptosis $(15,22)$. However, to date, whether or not $\mathrm{SH}$ affects the radiosensitivity of ESCC cells is unknown. Our study demonstrated that $\mathrm{SH}$ can increase the radiosensitivity of ESCC cells
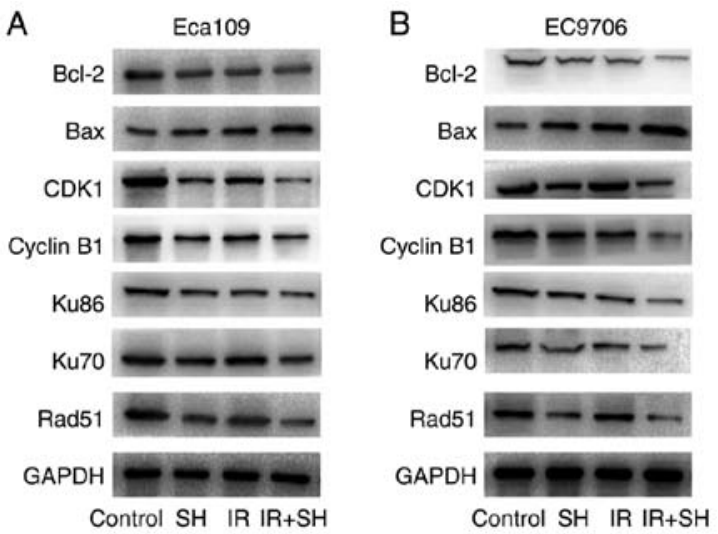

Figure 4. SH regulates the expression of Bax, Bcl-2, cyclin B1, CDK1, Ku-70, $\mathrm{Ku}-86$, and Rad51 in ESCC cells. SH markedly promoted Bax expression and inhibited $\mathrm{Ku}-70, \mathrm{Ku}-86, \mathrm{Bcl}-2$, cyclin B1, CDK1, and Rad51 expression. GAPDH was the internal control. (A) Eca109, (B) EC9706.
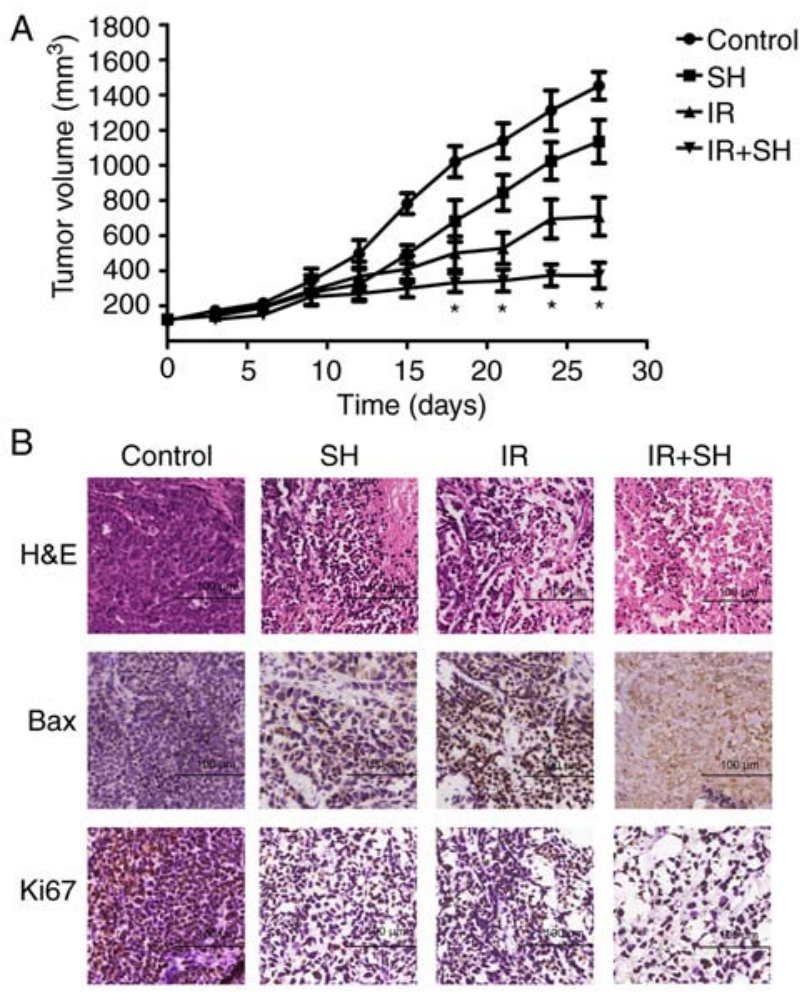

Figure 5. Combined treatment with $\mathrm{SH}$ and radiation effectively inhibits tumor growth in vivo. (A) The tumor volume was markedly smaller in the radiation $+\mathrm{SH}$ group than in the other three groups. Values are presented as mean \pm SEM ( $\mathrm{P}<0.05)$. (B) Immunohistochemical analysis demonstrated that the expression of Bax was significantly upregulated while Ki-67 was downregulated in the combined treatment group compared with the other three groups.

both in vitro and in vivo. We found that SH impeded ESCC cell proliferation in a time- and concentration-dependent manner and decreased the fraction of cells surviving after irradiation. Furthermore, radiosensitization of SH was related to $\mathrm{G} 2 / \mathrm{M}$ phase arrest through the downregulation of cyclin $\mathrm{B} 1$ and CDK1, apoptosis via the regulation of Bcl-2 and Bax expression, and downregulation of Ku86, Ku70, and Rad51 expression, which resulted in the inhibition of DNA-damage repair. 
The sensitivity of cells to radiation is related to the cell cycle phase. Cells in the specific cell cycle phases exhibit different degrees of radiosensitivity. In general, cells are most sensitive to irradiation in the $\mathrm{G} 2$ and $\mathrm{M}$ phases and are most radioresistant in the $\mathrm{S}$ phase. Cyclins and cyclin-dependent kinases (CDKs) are directly involved in the progression of the cell cycle (23). The cyclin B1/CDK1 complex plays an important role in G2/M phase transition (24). Numerous studies have demonstrated that many radiosensitizing agents, such as astaxanthin, sunitinib, and oxamate increase the cells in the G2/M cell cycle via inhibiting the expression of cyclin $\mathrm{B} 1$ and $\mathrm{CDK} 1$, which enhances the radiosensitivity of many malignant tumors $(7,25,26)$. Our study showed that radiation combined with $\mathrm{SH}$ could increase the number of Eca109 and EC9706 cells at G2/M phase, suggesting that $\mathrm{SH}$ arrested ESCC cells at G2/M phase and enhanced the lethal effects of irradiation on ESCC cells. The results of western blot showed that $\mathrm{SH}$ combined radiation significantly decreased the expression of cyclin B1 and CDK1, which arrested ESCC cells at G2/M phase.

Apoptosis is considered to be the primary process of cell death following radiotherapy (27). It is activated through different pathways, including both intrinsic (mitochondrial death pathway) and extrinsic pathways (death receptor pathway). The former pathway is regulated by the balance of anti-apoptotic and apoptogenic proteins, such as Bcl-2 and Bax, respectively (28). There is increasing evidence that the cell-apoptosis rate usually depends on the ratio of anti- and pro-apoptotic members, especially the ratio of Bcl-2 to Bax (29). $\mathrm{SH}$ has been demonstrated to promote apoptosis in various cancer cells (16,30). Other anticancer agents, such as oroxylin A and astaxanthin, have been shown to improve the sensitivity of radiation in cancer cells by promoting Bax expression and inhibiting $\mathrm{Bcl} 2$ expression $(6,25)$. These findings suggest that aberrations in $\mathrm{Bcl} 2$ and Bax expression may determine the cell fate and the anticancer effect of radiation therapy. In this study, ESCC cells were subjected to $\mathrm{SH}$ and radiation, both separately and in combination. Our results demonstrated that radiation and $\mathrm{SH}$ could synergistically increase the fraction of apoptotic cells. To investigate the mechanisms underpinning this synergistic effect, we performed western blot analysis. The results demonstrated that the radiation $+\mathrm{SH}$ group had increased Bax expression compared with other groups, in both Eca109 and EC9706 cells, which promoted cell apoptosis. In addition, Bcl-2 expression was considerably inhibited in the combination group. Furthermore, Bax expression in tumor tissues was upregulated in the combination group, as revealed by immunohistochemistry. Therefore, it seems that $\mathrm{Bcl} 2$ and Bax act as downstream signals mediating the effects of $\mathrm{SH}$ combined with radiation to induce apoptosis in ESCC cells.

The repair of DNA damage, especially double-strand breaks (DSBs), is a vital factor determining radiosensitivity. Non-homologous end joining (NHEJ) and homologous recombination (HR) are two major pathways of DNA-DSBs repair. RAD51, Ku86, and Ku70 are critical downstream factors of the NHEJ and HR pathways. Thus, the HR and NHEJ pathways are potential targets for radiosensitizing agents. Studies have revealed that cancer cells can be rendered more radiosensitive by inhibiting DNA-repair pathways. For example, the inhibition of RAD51 enhanced the radiosensitivity of non-small cell lung cancer cells, and the siRNA-mediated downregulation of
Ku80 expression can sensitize osteosarcoma cells to radiation probably via telomere length shortening $(31,32)$. In this study, western blot analysis indicated that SH sensitizes ESCC cells to radiation and impairs DNA repair via the downregulation of $\mathrm{Ku}-70, \mathrm{Ku}-86$, and Rad51 expression. Therefore, SH increased ESCC cell radiosensitivity by inhibiting DSB-repair pathways.

In addition, $\mathrm{SH}$ combined with radiation considerably inhibited the growth of tumor xenografts in vivo. Our data showed that the observed suppression in tumor growth was related with the inhibition of cell proliferation and the enhancement of apoptosis. Immunohistochemical analysis showed that in the combined treatment group, the expression of Bax was significantly higher while that of $\mathrm{Ki}-67$ was lower than the expressions in the other three groups.

In conclusion, our study is the first to demonstrate the effect of SH on the radiosensitivity of ESCC cells. Our findings provide evidence that $\mathrm{SH}$ is a promising radiosensitizer for improving the therapeutic efficacy of radiation in ESCC.

\section{Acknowledgements}

We are grateful to Medical Radiation Technologists of Radiation Oncology, the First Affiliated Hospital of Xi'an Jiaotong University for their significant contributions to this study.

\section{Competing interests}

The authors declare that they have no competing interests.

\section{References}

1. Torre LA, Bray F, Siegel RL, Ferlay J, Lortet-Tieulent J and Jemal A: Global cancer statistics, 2012. CA Cancer J Clin 65: 87-108, 2015.

2. Pennathur A, Gibson MK, Jobe BA and Luketich JD: Oesophageal carcinoma. Lancet 381: 400-412, 2013.

3. Siegel RL, Miller KD and Jemal A: Cancer statistics, 2017. CA Cancer J Clin 67: 7-30, 2017.

4. Enzinger PC and Mayer RJ: Esophageal cancer. N Engl J Med 349: 2241-2252, 2003.

5. Liu YM, Liu YK, Wang LW, Huang YC, Huang PI, Tsai TH and Chen YJ: The medicinal fungus Antrodia cinnamomea regulates DNA repair and enhances the radiosensitivity of human esophageal cancer cells. Onco Targets Ther 9: 6651-6661, 2016.

6. Tan C, Qian X, Ge Y, Yang B, Wang F, Guan Z and Cai J: Oroxylin a could be a promising radiosensitizer for esophageal squamous cell carcinoma by inducing $\mathrm{G} 2 / \mathrm{M}$ arrest and activating apoptosis. Pathol Oncol Res 23: 323-328, 2017.

7. Zhai X, Yang Y, Wan J, Zhu R and Wu Y: Inhibition of LDH-A by oxamate induces $\mathrm{G} 2 / \mathrm{M}$ arrest, apoptosis and increases radiosensitivity in nasopharyngeal carcinoma cells. Oncol Rep 30: 2983-2991, 2013

8. Kulkarni R, Thomas RA and Tucker JD: Expression of DNA repair and apoptosis genes in mitochondrial mutant and normal cells following exposure to ionizing radiation. Environ Mol Mutagen 52: 229-237, 2011.

9. Choudhury A, Zhao H, Jalali F, Al Rashid S, Ran J, Supiot S, Kiltie AE and Bristow RG: Targeting homologous recombination using imatinib results in enhanced tumor cell chemosensitivity and radiosensitivity. Mol Cancer Ther 8: 203-213, 2009.

10. Wang Y, Fang Y, Huang W, Zhou X, Wang M, Zhong B and Peng D: Effect of sinomenine on cytokine expression of macrophages and synoviocytes in adjuvant arthritis rats. J Ethnopharmacol 98: 37-43, 2005.

11. Qian L, Xu Z, Zhang W, Wilson B, Hong JS and Flood PM: Sinomenine, a natural dextrorotatory morphinan analog, is anti-inflammatory and neuroprotective through inhibition of microglial NADPH oxidase. J Neuroinflammation 4: 23, 2007. 
12. Kok TW, Yue PY, Mak NK, Fan TP, Liu L and Wong RN: The anti-angiogenic effect of sinomenine. Angiogenesis 8: 3-12, 2005.

13. Cheng Y, Zhang J, Hou W, Wang D, Li F, Zhang Y and Yuan F: Immunoregulatory effects of sinomenine on the T-bet/GATA-3 ratio and Th1/Th2 cytokine balance in the treatment of mesangial proliferative nephritis. Int Immunopharmacol 9: 894-899, 2009.

14. Yamasaki H: Pharmacology of sinomenine, an anti-rheumatic alkaloid from sinomenium acutum. Acta Med Okayama 30: 1-20, 1976.

15. Jiang T, Zhou L, Zhang W, Qu D, Xu X, Yang Y and Li S: Effects of sinomenine on proliferation and apoptosis in human lung cancer cell line NCI-H460 in vitro. Mol Med Rep 3: 51-56, 2010.

16. Lu XL, Zeng J, Chen YL, He PM, Wen MX, Ren MD, Hu YN, Lu GF and He S: Sinomenine hydrochloride inhibits human hepatocellular carcinoma cell growth in vitro and in vivo: Involvement of cell cycle arrest and apoptosis induction. Int J Oncol 42: 229-238, 2013.

17. Li X, Li P, Liu C, Ren Y, Tang X, Wang K and He J: Sinomenine hydrochloride inhibits breast cancer metastasis by attenuating inflammation-related epithelial-mesenchymal transition and cancer stemness. Oncotarget 8: 13560-13574, 2017.

18. Xie T, Ren HY, Lin HQ, Mao JP, Zhu T, Wang SD and Ye ZM: Sinomenine prevents metastasis of human osteosarcoma cells via $\mathrm{S}$ phase arrest and suppression of tumor-related neovascularization and osteolysis through the CXCR4-STAT3 pathway. Int J Oncol 48: 2098-2112, 2016.

19. Yang H, Yin P, Shi Z, Ma Y, Zhao C, Zheng J and Chen T: Sinomenine, a COX-2 inhibitor, induces cell cycle arrest and inhibits growth of human colon carcinoma cells in vitro and in vivo. Oncol Lett 11: 411-418, 2016.

20. Liao F, Yang Z, Lu X, Guo X and Dong W: Sinomenine sensitizes gastric cancer cells to 5-fluorouracil in vitro and in vivo. Oncol Lett 6: 1604-1610, 2013

21. Chou TC and Talalay P: Quantitative analysis of dose-effect relationships: The combined effects of multiple drugs or enzyme inhibitors. Adv Enzyme Regul 22: 27-55, 1984.

22. Jiang S, Gao Y, Hou W, Liu R, Qi X, Xu X, Li J, Bao Y, Zheng H and Hua B: Sinomenine inhibits A549 human lung cancer cell invasion by mediating the STAT3 signaling pathway. Oncol Lett 12: 1380-1386, 2016.

23. Pawlik TM and Keyomarsi K: Role of cell cycle in mediating sensitivity to radiotherapy. Int J Radiat Oncol Biol Phys 59: 928-942, 2004.
24. Lindqvist A, van Zon W, Karlsson Rosenthal C and Wolthuis RM: Cyclin B1-Cdk1 activation continues after centrosome separation to control mitotic progression. PLoS Biol 5: e123, 2007.

25. Qian X, Tan C, Yang B, Wang F, Ge Y, Guan Z and Cai J: Astaxanthin increases radiosensitivity in esophageal squamous cell carcinoma through inducing apoptosis and G2/M arrest. Dis Esophagus 30: 1-7, 2017

26. Ding YQ, Zhu HC, Chen XC, Sun XC, Yang X, Qin Q, Zhang H, Yang Y, Yang YH, Gao L, et al: Sunitinib modulates the radiosensitivity of esophageal squamous cell carcinoma cells in vitro. Dis Esophagus 29: 1144-1151, 2016.

27. Maier P, Hartmann L, Wenz F and Herskind C: Cellular pathways in response to ionizing radiation and their targetability for tumor radiosensitization. Int J Mol Sci 17: pii:E102, 2016.

28. Cory S, Huang DC and Adams JM: The Bcl-2 family: Roles in cell survival and oncogenesis. Oncogene 22: 8590-8607, 2003.

29. Naseri MH, Mahdavi M, Davoodi J, Tackallou SH Goudarzvand $\mathrm{M}$ and Neishabouri SH: Up regulation of Bax and down regulation of $\mathrm{Bcl} 2$ during 3-NC mediated apoptosis in human cancer cells. Cancer Cell Int 15: 55, 2015.

30. Li X, Wang K, Ren Y, Zhang L, Tang XJ, Zhang HM, Zhao CQ, Liu PJ, Zhang JM and He JJ: MAPK signaling mediates sinomenine hydrochloride-induced human breast cancer cell death via both reactive oxygen species-dependent and -independent pathways: An in vitro and in vivo study. Cell Death Dis 5: e1356, 2014.

31. Zhong X, Luo G, Zhou X, Luo W, Wu X, Zhong R, Wang Y, Xu F and Wang J: Rad51 in regulating the radiosensitivity of non-small cell lung cancer with different epidermal growth factor receptor mutation status. Thorac Cancer 7: 50-60, 2016.

32. Hu L, Wu QQ, Wang WB, Jiang HG, Yang L, Liu Y, Yu HJ, Xie CH, Zhou YF and Zhou FX: Suppression of Ku80 correlates with radiosensitivity and telomere shortening in the U2OS telomerase-negative osteosarcoma cell line. Asian Pac J Cancer Prev 14: 795-799, 2013.

This work is licensed under a Creative Commons Attribution-NonCommercial-NoDerivatives 4.0 International (CC BY-NC-ND 4.0) License. 